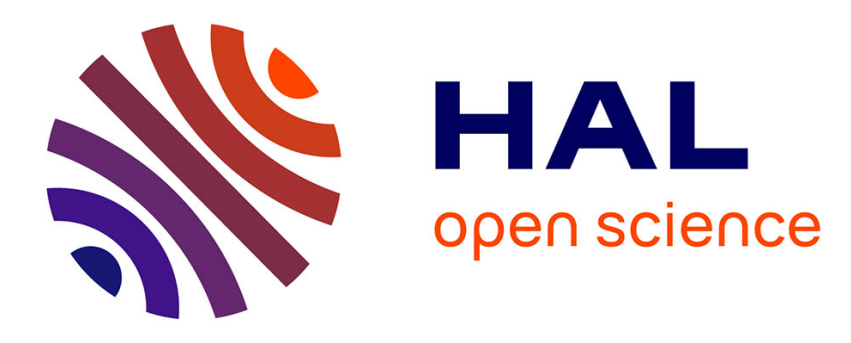

\title{
Stability of the HgS molecule and spectroscopy of its low lying electronic states \\ Gilberte Chambaud
}

\section{To cite this version:}

Gilberte Chambaud. Stability of the HgS molecule and spectroscopy of its low lying electronic states. Molecular Physics, 2007, 105 (09), pp.1207-1216. 10.1080/00268970701305501 . hal-00513090

\author{
HAL Id: hal-00513090 \\ https://hal.science/hal-00513090
}

Submitted on 1 Sep 2010

HAL is a multi-disciplinary open access archive for the deposit and dissemination of scientific research documents, whether they are published or not. The documents may come from teaching and research institutions in France or abroad, or from public or private research centers.
L'archive ouverte pluridisciplinaire $\mathbf{H A L}$, est destinée au dépôt et à la diffusion de documents scientifiques de niveau recherche, publiés ou non, émanant des établissements d'enseignement et de recherche français ou étrangers, des laboratoires publics ou privés. 


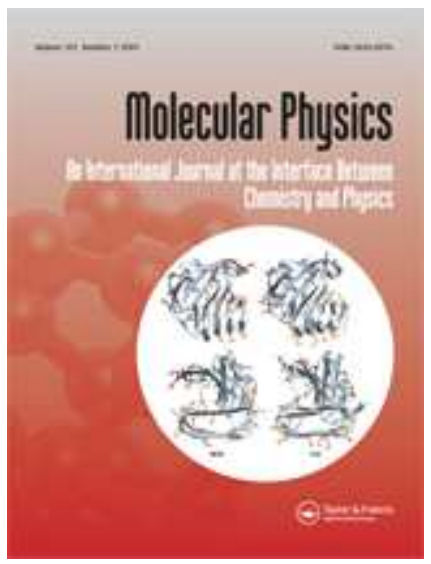

\section{Stability of the HgS molecule and spectroscopy of its low lying electronic states}

\begin{tabular}{|c|c|}
\hline Journal: & Molecular Physics \\
\hline Manuscript ID: & TMPH-2006-0115.R1 \\
\hline Manuscript Type: & Full Paper \\
\hline $\begin{array}{r}\text { Date Submitted by the } \\
\text { Author: }\end{array}$ & 17-Feb-2007 \\
\hline Complete List of Authors: & Gilberte, Chambaud; Université de Marne la Vallée \\
\hline Keywords: & $\begin{array}{l}\text { Electronic states, spin-orbit interaction, spectroscopy, } \\
\text { predissociation }\end{array}$ \\
\hline \multicolumn{2}{|c|}{$\begin{array}{l}\text { Note: The following files were submitted by the author for peer review, but cannot be converted } \\
\text { to PDF. You must view these files (e.g. movies) online. }\end{array}$} \\
\hline article-hgs-rev.tex & \\
\hline
\end{tabular}

\section{scholarONE" \\ Manuscript Central}




\title{
Stability of the HgS molecule and spectroscopy of its low lying electronic states
}

\author{
C. Cressiot ${ }^{a}$, M. Guitou ${ }^{a}$, A.O. Mitrushchenkov ${ }^{b}$ and G. Chambaud ${ }^{a}$. \\ ${ }^{a}$ Laboratoire de Chimie Théorique, \\ Université de Marne-la-Vallée, Champs sur Marne, France \\ ${ }^{b}$ Institut für Theoretische Chemie, Universität Stuttgart, Germany
}

\begin{abstract}
Large scale Multireference Configuration Interactions (MRCI) and energy consistent relativistic pseudopotential (for the $\mathrm{Hg}$ atom) have been used to investigate the electronic structure, stability and spectroscopy of the low lying electronic states of the $\mathrm{HgS}$ molecule. The relative position of the two lowest electronic states, $\mathrm{X}^{1} \Sigma^{+}$and $\mathrm{a}^{3} \Pi$, was found very sensitive to the quality of the basis set. Spin-orbit effects were taken into account leading to accurate spectroscopic data useful for the identification of the molecule. $T_{0}$ between the lowest components of the two states, $\mathrm{X}^{1} \Sigma_{0}^{+}$and $\mathrm{a}^{3} \Pi_{2}$, has been evaluated to be $0.142 \mathrm{eV}(3.5 \mathrm{kcal} / \mathrm{mol})$. Dipole moment functions were calculated for the lowest states; the rather large dipole moment of the $\mathrm{X}^{1} \Sigma^{+}$state makes possible the detection of vibrational transitions with a calculated $\omega_{e}$ equal to $364 \mathrm{~cm}^{-1}$. Transitions between the $\mathrm{X}^{1} \Sigma^{+}$and the $\mathrm{A}^{1} \Pi$ states are predicted in the far IR domain with a $T_{0}=5794 \mathrm{~cm}^{-1}$. The predissociation of the $\mathrm{X}^{1} \Sigma^{+}$and $\mathrm{A}^{1} \Pi$ states has been analysed and it has been shown that for the $\mathrm{X}^{1} \Sigma_{0}^{+}$state only the vibrational levels below $\mathrm{v}=11$ are stable; higher levels are predissociated by the $\mathrm{a}^{3} \Pi_{0}$ state. The effective dissociation energy of the $\mathrm{X}^{1} \Sigma_{0}^{+}$ state of $\mathrm{HgS}$ can thus be estimated to be $0.47 \mathrm{eV}(6.5 \mathrm{kcal} / \mathrm{mol})$. For the $\mathrm{A}^{1} \Pi$ state, the levels with $\mathrm{v}>8$ are predissociated by the dissociative $\mathrm{b}^{3} \Sigma^{-}$state.
\end{abstract}

\section{Introduction}

Solid mercury sulfide, $\mathrm{HgS}$, exists in several allotropic forms as mercury ore, the more common is a red cristal, known as cinnabar. The $\mathrm{HgS}$ molecule has however never been detected and was very scarcely studied. Calculations of $\mathrm{HgS}$ in aqueous solution were made by Tossel in 2001 [1] and more recently, the structure and energetics of gaseous oligomers and polymers of $(\mathrm{HgS})_{n}$ type [2] have been studied by the same author. Considering the very small dissociation energy $(4.0 \mathrm{kcal} / \mathrm{mol})$ of the valence isoelectronic $\mathrm{HgO}$ molecule, recently investigated by B.C. Shepler and K.A. Peterson [3], the stability of the $\mathrm{HgS}$ molecule is thus an open question that can be adressed using accurate electronic structure calculations. For this class of molecules, composed of oxygen (or sulfur) and a $\left(n s^{2}\right)$ metal, including molecular $\mathrm{ZnO}$ [4], and also valence isoelectronic compounds like $\mathrm{AlO}^{+}[5]$ and $\mathrm{AlS}^{+}[6]$, the two lowest electronic states, ${ }^{1} \Sigma^{+}$and ${ }^{3} \Pi$, are very close energetically and the nature of the ground state depends on the system. All these systems posses a close lying bound ${ }^{1} \Pi$ state which can lead 
to transitions with the ${ }^{1} \Sigma^{+}$state in the infrared or far infrared domain. The goal of the present study is thus to determine the exact nature of the ground state of $\mathrm{HgS}$, to estimate its dissociation energy and to provide data for a vibrational spectroscopy analysis.

The lowest covalent and ionic dissociation asymptotes of the diatomic $\mathrm{HgS}$ are reported in Table 1 and connected to the lowest molecular states of this system. As for the isoelectronic $\mathrm{HgO}$ system, the lowest asymptote has triplet character and correlates with one ${ }^{3} \Sigma^{-}$and one ${ }^{3} \Pi$ state, the second asymptote is singlet and correlates with one ${ }^{1} \Sigma^{+}$, one ${ }^{1} \Pi$ and one ${ }^{1} \Delta$ state. These asymptotes are separated by only 1.105 $\mathrm{eV}$ (energy averaged over the three fine structure components of the $\mathrm{S}\left({ }^{3} P\right)$ state[7]). The next asymptote corresponds to the excited ${ }^{1} S$ state of the $\mathrm{S}$ atom and gives rise to another ${ }^{1} \Sigma^{+}$state. The ionic dissociation asymptote, corresponding to $\mathrm{Hg}^{+}$and $\mathrm{S}^{-}$fragments, is calculated using the experimental ionisation energy of $\mathrm{Hg}$ and the electronic affinity of $\mathrm{S}$ (see footnotes of Table 1). It is located $8.36 \mathrm{eV}$ higher than the lowest one and gives rise to singlet and triplet states which are interacting with the lowest molecular states in the bonding region. The crossing distance $R_{c}$ between the covalent and ionic states can be roughly estimated by considering a variation as $-\frac{1}{R}$ of the potential energy of the ionic $\mathrm{Hg}^{+} \mathrm{S}^{-}$form and a quasi constant energy for the covalent states. Using the formula $\Delta E=-\frac{1}{R_{c}}$, where $\Delta E$ is the energy difference in atomic units between the asymptote of the covalent state and that of the ionic state, we find for the ${ }^{1} \Sigma^{+}$state, $\Delta E=-0.265$ a.u., and $R_{c}\left({ }^{1} \Sigma^{+}\right)=3.77 \mathrm{bohr}$, for the ${ }^{3} \Pi$, $\Delta E=-0.307$ a.u., and $R_{c}\left({ }^{3} \Pi\right)=3.25$ bohr. Even though this is a rough estimation of the interaction region, it is expected that the lowest states are getting ionic character in their molecular region, leading to multiconfigurational wavefunctions.

\section{Computational details}

\subsection{Basis sets}

The $\mathrm{Hg}$ atom presents large relativistic effects and for simplification its core electrons are usually described by a pseudo-potential including these effects. In the present study, we used for the $\mathrm{Hg}$ atom, the most recent energy-consistent ECP60MDF [8] effective core potentials, with 60 core electrons represented by a pseudo-potential and 20 outer electrons explicitely treated via the associated contracted basis sets [9] respectively corresponding to the schemes (11s 10p 9d 3f $2 \mathrm{~g}$ )/[6s 6p 5d 3f 2g], (15s 12p 11d 4f $3 \mathrm{~g}$ 2h)/[7s 7p 6d 4f 3g 2h] and (17s 14p 12d 5f 4g 3h 2i)/[8s 8p 7d 5f 4g 3h 2i] for the aug-cc-pVnZ $(n=3,4,5)$ basis sets. All the electrons of the sulfur atom were taken into account in the calculations, leading to a total number of 36 explicited electrons. For $\mathrm{S}$, the Dunning [10] correlation consistent aug-cc-pVnZ $(n=3,4,5)$ basis sets were used, with contraction schemes as follows : (16s 10p 3d 2f)/[6s 5p 3d 2f], (17s 12p 4d 3f $2 \mathrm{~g}) /[7 \mathrm{~s} 6 \mathrm{p} 4 \mathrm{~d} 3 \mathrm{f} 2 \mathrm{~g}]$ and $(21 \mathrm{~s} 13 \mathrm{p} 5 \mathrm{~d} 4 \mathrm{f} 3 \mathrm{~g}) /[8 \mathrm{~s} 7 \mathrm{p} 5 \mathrm{~d} 4 \mathrm{f} 3 \mathrm{~g}]$ respectively.

Since the relative position of the two lowest electronic states appears to be very sensitive to the basis set quality, as it has been also demonstrated for $\mathrm{HgO}$ [3], we used 
extrapolations towards complete basis sets for both atoms, following the two extrapolation formulae :

$$
\begin{gathered}
E(n)=E_{C B S}+B e^{-(n-1)}+C e^{-(n-1)^{2}} \\
E(n)=E_{C B S}+B / n^{3}
\end{gathered}
$$

Equation $1[11,12]$ is used with aug-cc-pVnZ $(\mathrm{n}=3,4,5)$ basis sets for both atoms (respectively noted AVTZ, AVQZ and AV5Z for $n=3,4,5$ later) and the method is referred as CBS(1), equation $2[13,14]$ is used only with the AVQZ and AV5Z basis sets for both atoms and is referred as CBS(2).

We have tested the influence of the correlation of the outer-core $5 s, 5 p$ orbitals of $\mathrm{Hg}$ with the cc-pwCV5Z basis set [15] for the $\mathrm{Hg}$ atom. The calculations of the ${ }^{3} \Pi$ state gives an $r_{e}$ increased by only 0.001 bohr compared to the AV5Z calculation. We have also tested the influence of a basis set extension with tight $d$ functions for $\mathrm{S}$, as recommended by Dunning et al. [16]. The changes on the value of $r_{e}$ were respectively $-0.012 \mathrm{bohr}$ and $+0.003 \mathrm{bohr}$, for the ${ }^{3} \Pi$ and ${ }^{1} \Sigma^{+}$states respectively and the influence on the values of $T_{e}$ and $\Delta \mathrm{E}_{\text {ass }}$, energy difference of the asymptote, were negligeable.

\subsection{Molecular structure calculations}

State-averaged Multi-Configuration-Self-Consistent-Field (MCSCF) calculations have been performed using 22 active molecular orbitals, constructed on the $5 s, 5 p, 5 d, 6 s$ and $6 p$ of $\mathrm{Hg}$, and $1 s, 2 s, 2 p, 3 s, 3 p$ of $\mathrm{S}$, to represent the wavefunction of the 36 electrons. Among these 22 orbitals, the 14 inner shells including the $5 d$ orbitals of $\mathrm{Hg}$ were not correlated in the MCSCF step. The resulting active space consisted in the $(7-10) \sigma$ and $(4-5) \pi$ orbitals. The MCSCF wavefunctions were taken as reference for the subsequent internally contracted Multi-Reference-Configuration-Interaction (MRCI) calculations[17]. The Davidson correction $[18,19](\mathrm{MRCI}+\mathrm{Q})$ which approximates the contribution of higher excitation terms, was added. Additional calculations were performed using the Coupled-Cluster $(\operatorname{CCSD}(\mathrm{T}))$ method for comparison. All electronic structure calculations were performed with the MOLPRO program package[20].

\section{Electronic structure of $\mathbf{H g S}$}

The state-averaged MCSCF calculations, using the AV5Z basis set for S and $\mathrm{Hg}$, were run with the lowest six electronic states correlated with the lowest three dissociation asymptotes, namely two ${ }^{1} \Sigma^{+}$, one ${ }^{1} \Delta$, one ${ }^{3} \Sigma^{-}$, one ${ }^{3} \Pi$ and one ${ }^{1} \Pi$ states. The subsequent MRCI calculations, using the MCSCF wavefunctions as references were run in each space and spin symmetry separately : for the ${ }^{1} \Sigma^{+}$and ${ }^{3} \Pi$ states, the MRCI steps involve 6120379392 and 1127322087 uncontracted configurations, respectively. At this stage, the ground state is found to be the ${ }^{1} \Sigma^{+}$state, contrary to the situation for 
$\mathrm{HgO}[3]$. The Figure 1 presents the potential energy curves (energies are given relative to the minimum of the ground state) of these electronic states at the MRCI level including the Davidson correction.

\subsection{Electronic wavefunctions}

The weights of the main configurations for the lowest electronic states at their respective equilibrium geometries, taken from the MCSCF wavefunctions, are reported in Table 2. The dominant characters of the external orbitals are as follows: the $7 \sigma$ orbital is mainly a combination of the $5 d_{z^{2}}$ of $\mathrm{Hg}$ (with some character of $6 s$ ) with the $3 \mathrm{~s}$ of $\mathrm{S}$, and it is doubly occupied in all the main configurations, the $4 \pi$ orbital corresponds mainly to the $3 p_{x}$ and $3 p_{y}$ of $\mathrm{S}$, the $8 \sigma$ and $9 \sigma$ are respectively the bounding and antibounding combinations of the $6 s$ orbital of $\mathrm{Hg}$ and the $3 p_{z}$ of S. Since the $6 p$ orbitals of $\mathrm{Hg}$ are not playing a major role in the leading configurations of the wavefunctions of these electronic states, the stability of these states can be roughly discussed in connection with the occupancy of the $8 \sigma$ and $9 \sigma$ orbitals: with two electrons in these two orbitals, the system is very stable, with three electrons the stability is less and with four electrons the system is unstable. For the ${ }^{1} \Sigma^{+}$state, the leading configuration is $\left(1 \sigma^{2} 2 \sigma^{2} 3 \sigma^{2} 4 \sigma^{2} 5 \sigma^{2} 6 \sigma^{2} 1 \delta^{4} 1 \pi^{4} 2 \pi^{4} 3 \pi^{4}\right) 7 \sigma^{2} 8 \sigma^{2} 4 \pi^{4}$, associated with a charge transfer from $\mathrm{Hg}$ towards S. The molecular orbitals in parentheses correspond essentially to atomic orbitals and are kept fully occupied in the MCSCF step. The leading configuration of both ${ }^{1} \Pi$ and ${ }^{3} \Pi$ states corresponds to a mono-excitation from the $4 \pi$ orbital to the antibounding $9 \sigma$ orbital, leading to a charge transfer from the $\mathrm{S}$ atom towards $\mathrm{Hg}$ and resulting in a less polarized bond than in the ${ }^{1} \Sigma^{+}$state. The leading configuration of both ${ }^{1} \Delta$ and ${ }^{3} \Sigma^{-}$states corresponds to a double excitation from the $4 \pi$ orbital to the antibounding $9 \sigma$ orbital, resulting in an inverse polarization of the bond and in unstable states. Many electronic states are found multiconfigurational with minor configurations involving the $6 p$ orbitals of $\mathrm{Hg}$ via the $8 \sigma, 9 \sigma$ and $5 \pi$ orbitals. We have thus preferred to apply the MRCI method throughout this study, instead of the Coupled-Cluster method, CCSD(T), based on a monoconfigurational expansion. However, some additional $\operatorname{CCSD}(\mathrm{T})$ calculations were performed to confirm the relative position of the two lowest electronic states.

\subsection{Dipole moment functions}

The dipole moment, as a function of the interatomic distance $\mathrm{R}$, is a useful tool to analyse the polarity of the molecule in the different electronic states and it is necessary to evaluate the intensities of the transition lines. These functions are calculated at the MRCI level for the lowest six electronic states. More states have been included in the calculations in order to have a better description of these functions in the regions of avoided crossings. Particularly important are the interactions involving the $\mathrm{X}^{1} \Sigma^{+}$and $2^{1} \Sigma^{+}$states around $3.5 \mathrm{bohr}$, the $2^{1} \Sigma^{+}$and $3^{1} \Sigma^{+}$states around $4.5 \mathrm{bohr}$, the $1^{1} \Delta$ and 
$2^{1} \Delta$ around $4.0 \mathrm{bohr}$ and the $1^{3} \Sigma^{-}$and $2^{3} \Sigma^{-}$states around 3 to 4 bohr. The dipole moment functions of the lowest six electronic states are represented in Figure 2 and the values at the equilibrium geometries of each of these states are given in Table 3. The sign convention for these quantities is such that a positive value corresponds here to a polarity $\mathrm{Hg}^{+} \mathrm{S}^{-}$. At large internuclear distances $(\mathrm{R}>7 \mathrm{bohr})$, the $\mathrm{X}^{1} \Sigma^{+}$state is covalent, with a quasi zero dipole moment. Due to interactions with higher states and particularly the ionic state going down from the $\left[\mathrm{Hg}^{+}+\mathrm{S}^{-}\right]$asymptote, the ionic character of this state increases when $\mathrm{R}$ decreases, with a maximum of the dipole moment function for R close to $4.5 \mathrm{bohr}$. In the bounding region, the state is found partially ionic $\mathrm{Hg}^{\delta+} \mathrm{S}^{\delta-}$, with a charge estimated to be $0.5 e$ according to the average slope of the dipole moment function. The ${ }^{1} \Pi$ and ${ }^{3} \Pi$ states correspond also to ionic structures of $\mathrm{Hg}^{\delta+} \mathrm{S}^{\delta-}$ type in the vicinity of their equilibrium geometries. The polarity is smaller in the ${ }^{3} \Pi$ state than in the ${ }^{1} \Pi$ because it is connected to an asymptote which is lower in energy and the interaction with the higher ionic state happens at shorter distances, closer to the repulsive wall. The dipole moment function of both the ${ }^{1} \Delta$ and the ${ }^{3} \Sigma^{-}$ states is negative in the molecular region, in agreement with an inversed polarity corresponding to $\mathrm{Hg}^{-} \mathrm{S}^{+}$. This inversed polarity is energetically unfavorable because of the large electron affinity of $\mathrm{S}(2.077 \mathrm{eV})$ [21], contrary to that of $\mathrm{Hg}$, when their ionization energies (10.36 eV and $10.43 \mathrm{eV}$, respectively [7]) are comparable. These two states are repulsive.

\section{Spectroscopic data of the three lowest bound elec- tronic states}

Spectroscopic data have been deduced from the potential energy functions of the three lowest bound electronic states with the NUMEROV method [22]. The results obtained with the potentials calculated with various methods and for different basis sets are reported in Table 4 . The results from CCSD(T) potentials give a slightly larger $\mathrm{r}_{e}$ (about $+0.015 \mathrm{bohr}$ ) and a slightly smaller $\omega_{e}$ (about $-6 \mathrm{~cm}^{-1}$ ) for the ${ }^{1} \Sigma^{+}$state compared to the values deduced from the MRCI+Q potentials. For the ${ }^{3} \Pi$ state, the values of $r_{e}$ and $\omega_{e}$ are larger by about $0.02 \mathrm{bohr}$ and $10 \mathrm{~cm}^{-1}$ respectively. In agreement with the electronic configurations discussed in section 2.1 , it is found that the equilibrium bond length of the ${ }^{1} \Sigma^{+}$is shorter than that of both the ${ }^{1} \Pi$ and ${ }^{3} \Pi$ states. The calculated dissociation energy value, relative to the adiabatic asymptote is also larger for the ${ }^{1} \Sigma^{+}$ state, $D_{e}=1.50 \mathrm{eV}$ than for the ${ }^{1} \Pi$ state, $D_{e}=0.79 \mathrm{eV}$ and for the ${ }^{3} \Pi$ state, $D_{e}=$ $0.23 \mathrm{eV}$ (MRCI+Q values calculated with the AV5Z basis). The other states correlated with the first two asymptotes are repulsive. The energy difference between the lowest two asymptotes is calculated (AV5Z) to be $1.10 \mathrm{eV}$, which compares very well with the experimental averaged value of $1.105 \mathrm{eV}$ [7].

A vibronic transition of medium intensity can be expected between the ${ }^{1} \Sigma^{+}$and ${ }^{1} \Pi$ bound states due to a transition dipole moment between these two states calculated to 
be 0.415 a.u. (MRCI+Q) at the equilibrium geometry of the ${ }^{1} \Sigma^{+}$state. This transition is expected in the far infra-red domain, according to the calculated energy difference $T_{0}=0.718 \mathrm{eV}\left(5794 \mathrm{~cm}^{-1}\right)$.

\section{Relative position of the $1^{1} \Sigma^{+}$and $1^{3} \Pi$ states}

\subsection{Basis set effects}

It is well known that the correct relative position of singlet and triplet states on one hand, and of $\Sigma$ and $\Pi$ states on the other hand is accessible only with highly correlated wavefunctions; the better the calculation, the lower is the ${ }^{1} \Sigma^{+}$state relative to the ${ }^{3} \Pi$ state. In the present study, we used a large scale Configuration Interaction method, and the possible improvement stems on the quality of the linear space accessible via the atomic and molecular basis set. The results are compared with $\operatorname{CCSD}(\mathrm{T})$ calculations with the same basis sets. The MRCI calculations have been performed separately for the ${ }^{1} \Sigma^{+}$and the ${ }^{3} \Pi$ states. The Figure 3 presents the relative energies of the two states, calculated at the MRCI+Q level of theory, with different basis sets. On this figure, we can see that already with the AVTZ basis set $(n=3)$, the minimum of the ${ }^{1} \Sigma^{+}$state is below that of the ${ }^{3} \Pi$ state. The energetic gap between the lowest two states increases with the size of the basis set, indicating that the ${ }^{1} \Sigma^{+}$is actually the ground state, contrary to $\mathrm{HgO}[3]$ where the ${ }^{3} \Pi$ state was found lower than the ${ }^{1} \Sigma^{+}$ state with $T_{e}=0.018 \mathrm{eV}(0.46 \mathrm{kcal} / \mathrm{mol})$ at this stage of the calculation. For $\mathrm{HgS}$, the best estimation of $T_{e}$ is $0.193 \mathrm{eV}(4.84 \mathrm{kcal} / \mathrm{mol})$ with MRCI+Q calculations and 0.150 $\mathrm{eV}$ with $\operatorname{CCSD}(\mathrm{T})$ calculations. The corresponding data are given in Table 4.

\subsection{Spin-orbit interactions}

In the asymptotic region, the ${ }^{1} \Sigma^{+}$state is correlated with $\left[\operatorname{Hg}\left({ }^{1} S\right)+\mathrm{S}\left({ }^{1} D\right)\right]$ and there is neither spin-orbit splitting for $\mathrm{Hg}$ nor for $\mathrm{S}$; the five sub-levels of the $\mathrm{S}\left({ }^{1} D_{2}\right)$ state corresponding to $\mathrm{m}_{J}= \pm 2, \pm 1$ and 0 are degenerated and give rise to the ${ }^{1} \Delta,{ }^{1} \Pi$ and ${ }^{1} \Sigma^{+}$states, respectively. The ${ }^{1} \Sigma^{+}$state corresponds to $\mathrm{m}_{J}=0$ and a total quantum number $\Omega=0$.

The ${ }^{3} \Pi$ state is correlated, in the asymptotic region, to $\left[\mathrm{Hg}\left({ }^{1} S\right)+\mathrm{S}\left({ }^{3} P\right)\right]$, and the degeneracy of the $\mathrm{S}\left({ }^{3} P\right)$ is raised by spin-orbit interaction, giving three levels with $J=$ 2,1 and 0 with respective experimental energies equal to $0 ; 396.8$ and $573.6 \mathrm{~cm}^{-1}$ [7]. This is an inverted order of $J$ levels as expected for $\mathrm{S}$, with a more than half full $p$ shell. The spacing between the three states is not exactly regular ( $2 A$ and $A$, where $A$ is the spin-orbit constant) because the $J=2$ level interacts with the $\mathrm{S}\left({ }^{1} D_{2}\right)$ state, and the $J$ $=0$ level interacts with the $\mathrm{S}\left({ }^{1} S_{0}\right)$ state. The three fine structure levels of the $\mathrm{S}\left({ }^{3} \mathrm{P}\right)$ give rise to the three $\Omega=2,1$ and 0 components of the ${ }^{3} \Pi$ state which are separated, at infinite internuclear separation, by the atomic energy differences mentioned above. Among these three, the $\Omega=0$ state (component $0^{+}$) interacts with the ${ }^{1} \Sigma^{+}$state. 
For the ${ }^{3} P,{ }^{1} D$ and ${ }^{1} S$ states of the $\mathrm{S}$ atom, corresponding to the same electronic configuration $\left(1 s^{2}, 2 s^{2}, 2 p^{6}, 3 s^{2}, 3 p^{4}\right)$, we have calculated the Breit-Pauli spin-orbit matrix elements, with the AV5Z basis set and MRCI wavefunctions, using the MOLPRO code[20]. The $A$ constant has been evaluated to be $182.3 \mathrm{~cm}^{-1}$, approximately $13 \mathrm{~cm}^{-1}$ smaller than the experimental value, the ${ }^{3} P_{2} /{ }^{1} D_{2}$ interaction lowers the ${ }^{3} P_{2}$ by $6 \mathrm{~cm}^{-1}$ and the ${ }^{3} P_{0} /{ }^{1} S_{0}$ interaction lowers the ${ }^{3} P_{0}$ by $12 \mathrm{~cm}^{-1}$.

Using the spin-orbit splitting, we can raise the degeneracy of the fine structure components of the lowest asymptote. For the $\mathrm{S}\left({ }^{3} P_{0}\right)$ components, the position of the asymptote must be raised by $378 \mathrm{~cm}^{-1}(0.047 \mathrm{eV})$ with respect to the calculated energy of this asymptote ; for the $\mathrm{S}\left({ }^{3} P_{1}\right)$ component, the asymptote is raised by 195 $\mathrm{cm}^{-1}(0.024 \mathrm{eV})$, for the $\mathrm{S}\left({ }^{3} P_{2}\right)$ component, the asymptote is lowered by $201 \mathrm{~cm}^{-1}$ $(0.025 \mathrm{eV})$. Hence the spin-orbit splitting of the ${ }^{3} \Pi$ state influences the relative position of the ${ }^{3} \Pi$ and ${ }^{1} \Sigma^{+}$states because this latter is not shifted in the asymptotic region.

\subsection{Variations of the spin-orbit interactions with $R$}

To calculate the dependence of spin-orbit matrix elements on the distance, we used a combined pseudo-potential+Breit-Pauli approach. The Breit-Pauli one- and twoelectron spin-orbit integrals were kept only for the $\mathrm{S}$ atom, while for the $\mathrm{Hg}$ atom the pseudo-potential one-electron integrals were used. We have checked that the accuracy of such a combined one-center approximation is better than $1 \mathrm{~cm}^{-1}$ at short distances and better than $0.1 \mathrm{~cm}^{-1}$ close to dissociation. The evolution of the spinorbit matrix elements involving the lowest electronic ${ }^{1} \Sigma_{0}^{+},{ }^{3} \Pi_{0,1},{ }^{1} \Pi_{1}$ and ${ }^{3} \Sigma_{1}^{-}$states are depicted on Figure 4. At short internuclear distance, the electrons perceive the large spin-orbit interaction of $\mathrm{Hg}$, resulting in a strong increase of the matrix elements. The energy of the ${ }^{3} \Pi_{0}$ state including spin-orbit is evaluated by shifting the calculated ${ }^{3} \Pi$ by $2 \times\left\langle{ }^{3} \Pi\left|\mathrm{H}_{s o}\right|^{3} \Pi\right\rangle$. The inclusion of this spin-orbit interaction increases the $T_{e}(\Omega=0)$ value by $610 \mathrm{~cm}^{-1}$. We estimated, at a MCSCF level, that the effect of the second-order spin-orbit interaction from higher lying states (excluding the five lowest) is negligible at all relevant distances. We can confirm that, as for $\mathrm{HgO}$, the nature of the ground fine structure component $(\Omega=0)$ is changing from a dominant ${ }^{1} \Sigma^{+}$configuration to a ${ }^{3} \Pi$ for $\mathrm{R}>4.67 \mathrm{bohr}$ if we adopt a spin-orbit adiabatic representation of the state. We prefer however to describe the states in a spin-orbit diabatic way and to discuss the stability in terms of the reactivity of the vibrational states. Equivalent corrections are made for the two other components of the ${ }^{3} \Pi$ state, and finally the global $T_{e}$ (between ${ }^{1} \Sigma_{0}$ and ${ }^{3} \Pi_{2}$ ) is reduced by $303 \mathrm{~cm}^{-1}$. The spectroscopic constants for each component of the ${ }^{3} \Pi$ state corrected by these spin-orbit interactions, are also presented in Table 4. 


\subsection{Effect of the zero vibrational level}

The harmonic wavenumber $\omega_{e}$ of the $\mathrm{X}^{1} \Sigma^{+}$state is calculated to be $364 \mathrm{~cm}^{-1}$ (potential obtained from MRCI+Q and CBS(2) extrapolation), whereas it is only $150 \mathrm{~cm}^{-1}$ for the ${ }^{3} \Pi$ state (see Table 4). Correcting from the vibrational zero energy level, gives a $T_{0}=T_{e}-0.013 \mathrm{eV}=0.142 \mathrm{eV}(3.5 \mathrm{kcal} / \mathrm{mol})$ between the ${ }^{1} \Sigma_{0}$ and ${ }^{3} \Pi_{2}$ states, so that the conclusion on the nature of the ground state is unchanged.

\section{Predissociation of the $\mathbf{X}^{1} \Sigma^{+}$and of the $A^{1} \Pi$}

For the following discussion, we used the potentials obtained at the MRCI+Q level and with CBS(2) extrapolation. The $\mathrm{X}^{1} \Sigma_{0}^{+}$and the $\mathrm{a}^{3} \Pi_{0}$ states are crossing for an internuclear distance of about $4.72 \mathrm{bohr}$, at an energy of $2222 \mathrm{~cm}^{-1}$ above the minimum of the ground state. These two states are interacting via spin-orbit interactions, with particularly large electronic interactions at short internuclear distances as shown in Figure 4. The global spin-orbit effect between the vibrational levels of these two electronic states is particularly important in the region of the crossing and above it. The spin-orbit interaction term between two vibronic states can be written as follows :

$$
S O_{v v^{\prime}}=\left\langle\Psi^{s e} \Psi_{v}^{e}\left|\hat{H_{S O}}\right| \Psi^{s^{\prime} e^{\prime}} \Psi_{v^{\prime}}^{e^{\prime}}\right\rangle=\left\langle\Psi_{v}^{e} \mid \Psi_{v^{\prime}}^{e^{\prime}}\right\rangle\left\langle\Psi^{s e}\left|\hat{H_{S O}}\right| \Psi^{s^{\prime} e^{\prime}}\right\rangle
$$

In the right hand side expression, the first integral is the Franck-Condon factor, the second one is the spin-orbit integral evaluated above. From this expression, it is clear that the spin-orbit effect within the fine structure components of the $a^{3} \Pi$ state is mainly represented by the electronic spin-orbit effect since the Franck-Condon factor equals 1 for a given $\mathrm{v}$ value. This property has been used to describe the vibrational levels of this state in the previous section. The description of the vibronic interaction between two different electronic states must include the role of the Franck-Condon factor. Consequently, only the vibrational states of the $\mathrm{X}^{1} \Sigma^{+}$located above the crossing will be perturbed by those of the ${ }^{3} \Pi$ state and all the vibrational states with energies higher than $3797 \mathrm{~cm}^{-1}$ above the minimum of the ground state (corresponding to the dissociation limit of the ${ }^{3} \Pi_{0}$ state) are predissociated. We calculated the energies of the lowest vibrational levels of the $\mathrm{X}^{1} \Sigma^{+}$state and we found that the number of non-predissociated vibrational levels is equal to 11 . The calculated energy levels of the lowest vibrational states of the involved electronic states are given in Table 5. Some vibrational levels of the ${ }^{1} \Sigma_{0}^{+}$state are calculated quasi-resonant with vibrational states of the $\mathrm{a}^{3} \Pi_{0}$ and it can be expected that large perturbations occur.

Similarly the $\mathrm{A}^{1} \Pi_{1}$ state is predissociated by the repulsive ${ }^{3} \Sigma_{1}^{-}$, correlated with the lowest dissociation asymptote, for vibrational levels immediately above the crossing of both states, occuring at $\mathrm{R}=5.15 \mathrm{bohr}$ and $\Delta \mathrm{E}=2357 \mathrm{~cm}^{-1}$ above the minimum of the ${ }^{1} \Pi_{1}$ state (values calculated from the CBS(2) extrapolation - PEFs of the ${ }^{1} \Pi_{1}$ and ${ }^{3} \Sigma_{1}^{-}$ calculated separately). The efficiency of this predissociation is equivalent to that of the 
$\mathrm{X}^{1} \Sigma^{+}$ground state by the ${ }^{3} \Pi_{0}$ because the spin-orbit interaction is of the same order, see Figure 4. Calculations of the lowest vibrational levels of the ${ }^{1} \Pi_{1}$ state indicate that this happens for $\mathrm{v}>8$.

It is possible to observe transitions between the vibrational levels of $\mathrm{X}$ and $\mathrm{A}$ states. The $T_{0}$ values are given in Table 5 for the lowest spin-electronic states, relative to the $\mathrm{v}=0$ value of the ground electronic state. Higher vibrational levels are also listed in this Table and energies of possible transitions can be deduced from it.

\section{Comparison with the $\mathrm{HgO}$ molecule and other va- lence isoelectronic molecules}

It is a general characteristic of all the molecules of this family to have almost degenerated ${ }^{3} \Pi$ and ${ }^{1} \Sigma^{+}$ground states, the ${ }^{3} \Pi$ state, correlated with the lowest dissociation asymptote being weakly bound contrary to the ${ }^{1} \Sigma^{+}$state correlated with the second dissociation asymptote.

For the $\mathrm{HgO}$ molecule, it has been found that the ${ }^{3} \Pi$ state is lower than the ${ }^{1} \Sigma^{+}$state by only $0.46 \mathrm{kcal} / \mathrm{mol}$. Two competiting effects can tentatively explain this small difference with the $\mathrm{HgS}$ system : first, the fact that the ${ }^{1} D$ state of oxygen is higher than the ${ }^{1} D$ state of sulfur with respect to their ${ }^{3} P$ states $\left(\Delta \mathrm{E}={ }^{1} D(\mathrm{O})-{ }^{1} D(\mathrm{~S})=0.82 \mathrm{eV}\right)$ implies that the adiabatic asymptote of the ${ }^{1} \Sigma^{+}$state of $\mathrm{HgO}$ is higher than the $\mathrm{HgS}$ one, leading to a global raise of the ${ }^{1} \Sigma^{+}$potential curve of $\mathrm{HgO}$ compared to that of $\mathrm{HgS}$. Second, the fact that the oxygen atom is smaller than sulfur results in a shorter bond length and a larger dissociation energy of the ${ }^{1} \Sigma^{+}$state (for $\mathrm{HgO}$, the equilibrium distances of the ${ }^{1} \Sigma^{+}$and ${ }^{3} \Pi$ states are $3.6 \mathrm{bohr}$ and $4.2 \mathrm{bohr}$, respectively [3] to be compared with 4.3 and $4.9 \mathrm{bohr}$, respectively for $\mathrm{HgS}$ ). The energy difference of the ${ }^{1} D$ states is the dominant effect in this case.

The situation is different for the valence isoelectronic $\mathrm{AlO}^{+}[5]$ and $\mathrm{AlS}^{+}[6]$ systems because the bonds are much more ionic and the bond lengths are shorter. The $\mathrm{X}^{1} \Sigma^{+}$ state is $720 \mathrm{~cm}^{-1}$ lower than the $\mathrm{a}^{3} \Pi$ state for $\mathrm{AlO}^{+}$, whereas it is the $\mathrm{X}^{3} \Pi$ state which is $1500 \mathrm{~cm}^{-1}$ lower than the $\mathrm{a}^{1} \Sigma^{+}$state for $\mathrm{AlS}^{+}$. The smaller size of the oxygen atom is the dominant effect in this case.

\section{Conclusions}

An accurate study of the $\mathrm{HgS}$ molecule with extended basis sets and highly correlated wavefunctions was necessary to determine the relative position of the $\mathrm{X}^{1} \Sigma^{+}$and $\mathrm{a}^{3} \Pi$ 
states. Even though MRCI+Q and $\operatorname{CCSD}(\mathrm{T})$ calculations do not give exactly the same values for the e,ergy differences, the conclusion on the relative position of the two lowest electronic states is the same. Spin-orbit effects and zero-vibrational corrections were introduced in the present analysis. Spectroscopic data werededuced for the lowest three electronic states allowing an experimental identification of the $\mathrm{HgS}$ molecule. The predissociation of the $\mathrm{X}^{1} \Sigma^{+}$state by the $\mathrm{a}^{3} \Pi$ state and that of the $\mathrm{A}^{1} \Pi$ state by the $\mathrm{b}^{3} \Sigma^{-}$state have been investigated.

\section{References}

[1] J.A. Tossel J. Phys. Chem. A 105, 935 (2001).

[2] J.A. Tossel J. Phys. Chem. A 110, 2571 (2006).

[3] B. C. Shepler and K. A. Peterson J. Phys. Chem. A 107, 1783 (2003).

[4] C. W. Bauschlicher Jr. and S.R. Langhoff Chem. Phys. Lett. 126, 163 (1986).

[5] G. Chambaud, P. Rosmus, M. L. Senent and P. Palmieri Molecular Physics 92, 399 (1997).

[6] M. Guichemerre and G. Chambaud J. Phys. Chem. A 104, 2105 (2000).

[7] C. E. Moore Atomic Energy Levels, Vol.1-3, May 1958.

[8] D. Figgen, G. Rauhaut, M. Dolg and H. Stoll Chem. Phys. 311, 227 (2005).

[9] K. A. Peterson and C. Puzzarini Theor. Chem. Acc. 114, 283 (2005).

[10] D.E. Woon and T.H. Dunning Jr. J. Chem. Phys. 98, 1358 (1993).

[11] K. A. Peterson, D. E. Woon and T. H. Dunning Jr. J. Chem. Phys. 100, 7410 (1994).

[12] D. Feller and K. A. Peterson J. Chem. Phys. 110, 8384 (1999).

[13] T. Helgaker, W. Klopper, H. Koch and J. Noga J. Chem. Phys. 106, 9639 (1997).

[14] A. Halkier, T. Helgaker, P. Jørgensen, W. Klopper, H. Koch, J. Olsen and A. K. Wilson Chem. Phys. Lett. 286, 243 (1998).

[15] K. A. Peterson and T.H. Dunning, Jr. J. Chem. Phys. 117, 10548 (2002).

[16] T.H. Dunning, Jr., K. A. Peterson and A. K. Wilson J. Chem. Phys. 114, 9244 (2001).

[17] H.-J. Werner and P.J. Knowles J. Chem. Phys. 89, 5803 (1988); P.J. Knowles and H.-J. Werner Chem. Phys. Lett. 145, 514 (1988).

[18] S. R. Langhoff and E. R. Davidson Int. J. Quantum Chem., 8, 61 (1974). 
[19] M. R. A. Blomberg and P. E. M. Siegbahn J. Chem. Phys., 78, 5682 (1983).

[20] MOLPRO, version 2006.1, a package of ab initio programs, H.-J. Werner, P. J. Knowles, R. Lindh, F. R. Manby, M. Schütz, and others , see http://www.molpro.net.

[21] J. Emsley, The Elements, Second Edition, Oxford University Press (1989).

[22] The code NUMEROV used in the present application was written by J. Senekowitsch et al., Johan Wolfgang Goethe Universität, Frankfurt-am-Main, Germany.

[23] Lide, D.R. (Editor), Ionization potentials of atoms and atomic ions in Handbook of Chem. and Phys., 10 (1992).

\section{Figures caption}

Figure 1: Potential energy curves, calculated at the MRCI+Q level and AV5Z basis sets, of the electronic states of $\mathrm{HgS}$ correlated to the lowest three asymptotes.

Figure 2: Dipole moment functions, calculated at the MRCI level and AV5Z basis sets, of the low lying electronic states of $\mathrm{HgS}$.

Figure 3 : Potential energy curves ${ }^{a}$ of the ${ }^{1} \Sigma^{+}$and ${ }^{3} \Pi$ states of $\mathrm{HgS}$ for different basis sets and with CBS extrapolations.

Figure 4 : Evolution of the spin-orbit matrix elements $\left(\mathrm{in}^{-1}\right.$ ) between the low lying electronic states ${ }^{a}$.

Figure 5 : Potential energy curves of the ${ }^{1} \Sigma_{\Omega=0}^{+}$and ${ }^{3} \Pi_{\Omega=0,1,2}$ states of $\operatorname{HgS}(\mathrm{CBS}(2)$ extrapolation including spin-orbit interactions). 
Table 1: Electronic molecular states of the $\mathrm{HgS}$ molecule correlated to the lowest states of the atomic fragments.

\begin{tabular}{c|c|c}
\hline \hline Atomic states & Energies $^{(a)} / \mathrm{eV}$ & Molecular states \\
\hline $\mathrm{Hg}\left({ }^{1} S_{g}\right)+\mathrm{S}\left({ }^{3} P_{g}\right)$ & 0 & ${ }^{3} \Sigma^{-},{ }^{3} \Pi$ \\
$\mathrm{Hg}\left({ }^{1} S_{g}\right)+\mathrm{S}\left({ }^{1} D_{g}\right)$ & 1.105 & ${ }^{1} \Sigma^{+},{ }^{1} \Pi,{ }^{1} \Delta$ \\
$\mathrm{Hg}\left({ }^{1} S_{g}\right)+\mathrm{S}\left({ }^{1} S_{g}\right)$ & 2.710 & ${ }^{1} \Sigma^{+}$ \\
$\mathrm{Hg}\left({ }^{3} P_{u}\right)+\mathrm{S}\left({ }^{3} P_{g}\right)$ & 5.005 & ${ }^{3} \Sigma^{+},{ }^{3} \Sigma^{-}(2),{ }^{3} \Pi(2),{ }^{3} \Delta$, \\
& & ${ }^{1} \Sigma^{+},{ }^{1} \Sigma^{-}(2),{ }^{1} \Pi(2),{ }^{1} \Delta$ \\
$\mathrm{Hg}\left({ }^{3} P_{u}\right)+\mathrm{S}\left({ }^{1} D_{g}\right)$ & 6.110 & $3 \Sigma^{+}(2),{ }^{3} \Sigma^{-},{ }^{3} \Pi(3),{ }^{3} \Delta(3)$, \\
& & ${ }^{1} \Sigma^{+}(2),{ }^{1} \Sigma^{-},{ }^{1} \Pi(3),{ }^{1} \Delta(3)$ \\
$\ldots$ & $\ldots . .$. \\
$\mathrm{Hg}^{+}\left({ }^{2} S_{g}\right)+\mathrm{S}^{-}\left({ }^{2} P_{u}\right)$ & 8.360 & ${ }^{3} \Sigma^{+},{ }^{3} \Pi,{ }^{1} \Sigma^{+},{ }^{1} \Pi$ \\
\hline \hline
\end{tabular}

(a): From C. Moore [7], for the S $\left({ }^{3} P_{g}\right)$ state and for the $\mathrm{Hg}\left({ }^{3} P_{u}\right)$ state, the values are averaged over the three fine structure levels; Ionization potential of $\mathrm{Hg}: 10.437 \mathrm{eV}$ [23]; Experimental electronic affinity of $\mathrm{S}$ : $2.077 \mathrm{eV}[21]$

Table 2: Dominant configurations in the MCSCF wavefunctions of the lowest electronic states ${ }^{a}$.

\begin{tabular}{c|c|c}
\hline \hline States & Configuration $^{b}$ & Coefficient \\
\hline${ }^{1} \Sigma^{+}$ & $\ldots 7 \sigma^{2} 8 \sigma^{2} 4 \pi^{4}$ & 0.8897960 \\
${ }^{1} \Pi$ & $\ldots 7 \sigma^{2} 8 \sigma^{2} 9 \sigma^{1} 4 \pi^{3}$ & 0.6745726 \\
${ }^{3} \Pi$ & $\ldots 7 \sigma^{2} 8 \sigma^{2} 9 \sigma^{1} 4 \pi^{3}$ & 0.9744153 \\
${ }^{1} \Delta$ & $\ldots 7 \sigma^{2} 8 \sigma^{2} 9 \sigma^{2} 4 \pi^{2}$ & 0.6639346 \\
${ }^{3} \Sigma^{-}$ & $\ldots 7 \sigma^{2} 8 \sigma^{2} 9 \sigma^{2} 4 \pi^{2}$ & 0.9764940 \\
$2^{1} \Sigma^{+}$ & $\ldots 7 \sigma^{2} 8 \sigma^{2} 9 \sigma^{2} 4 \pi^{2}$ & 0.6388155 \\
\hline \hline
\end{tabular}

(a): Configurations at the equilibrium geometry of the states. For repulsive states, the configurations are taken at the equilibrium geometry of the ${ }^{1} \Sigma^{+}$state.

(b): the dots represent the orbitals frozen in the MCSCF step: $1 \sigma^{2} 2 \sigma^{2} 3 \sigma^{2} 4 \sigma^{2} 5 \sigma^{2} 6 \sigma^{2} 1 \delta^{4} 1 \pi^{4} 2 \pi^{4}$ $3 \pi^{4}$.

Table 3: Dipole moments (MRCI) at the equilibrium geometry ${ }^{a}$ of the states.

\begin{tabular}{c|cccccc}
\hline \hline States & ${ }^{1} \Sigma^{+}$ & ${ }^{1} \Pi$ & ${ }^{3} \Pi$ & ${ }^{1} \Delta$ & ${ }^{3} \Sigma^{-}$ & $2^{1} \Sigma^{+}$ \\
\hline$r_{e} /$ bohr & 4.2 & 4.5 & 4.8 & & & 5.7 \\
\hline$\mu_{e}$ /a.u. & 1.89 & 0.87 & 0.72 & -1.38 & -1.43 & -0.08 \\
\hline \hline
\end{tabular}

(a): The dipole moments are calculated at the equilibrium geometry of each bonding states. For repulsive states, the dipole are given at the equilibrium geometry of the ${ }^{1} \Sigma^{+}$state. 
Table 4: Spectroscopic constants of the $\mathrm{X}^{1} \Sigma^{+}, \mathrm{a}^{3} \Pi$ and $\mathrm{A}^{1} \Pi$ states for different basis sets.

\begin{tabular}{|c|c|c|c|c|c|c|c|}
\hline & Method & $\mathrm{r}_{e} / \mathrm{bohr}$ & $\omega_{e}^{a} / \mathrm{cm}^{-1}$ & $\bar{\omega}_{\omega_{e} x_{e} / \mathrm{cm}^{-1}}$ & $\mathrm{D}_{e} / \mathrm{eV}$ & $\mathrm{T}_{e}^{b} / \mathrm{eV}$ & $\Delta E_{\text {ass. }}^{c} / \mathrm{eV}$ \\
\hline & & \multicolumn{6}{|c|}{${ }^{1} \Sigma^{+}$state } \\
\hline \multirow[t]{2}{*}{ AVTZ } & MRCI+Q & 4.271 & 355.5 & 2.6 & 1.408 & & \\
\hline & $\operatorname{CCSD}(\mathrm{T})$ & 4.287 & 348.9 & 1.9 & & & \\
\hline \multirow[t]{2}{*}{ AVQZ } & $\mathrm{MRCI}+\mathrm{Q}$ & 4.253 & 360.5 & 2.4 & 1.483 & & \\
\hline & $\operatorname{CCSD}(\mathrm{T})$ & 4.267 & 355.0 & 2.1 & & & \\
\hline \multirow[t]{2}{*}{ AV5Z } & $\mathrm{MRCI}+\mathrm{Q}$ & 4.246 & 362.0 & 2.3 & 1.504 & & \\
\hline & $\operatorname{CCSD}(\mathrm{T})$ & 4.259 & 356.8 & 2.3 & & & \\
\hline \multirow[t]{2}{*}{ CBS(1) } & MRCI+Q & 4.241 & 362.9 & 2.3 & 1.516 & & \\
\hline & $\operatorname{CCSD}(\mathrm{T})$ & 4.255 & 357.8 & 2.3 & & & \\
\hline \multirow[t]{3}{*}{ CBS(2) } & MRCI+Q & 4.237 & 363.6 & 2.2 & 1.526 & & \\
\hline & $\operatorname{CCSD}(\mathrm{T})$ & 4.251 & 358.7 & 2.4 & & & \\
\hline & & & & ${ }^{3} \Pi s$ & & & \\
\hline \multirow[t]{2}{*}{ AVTZ } & MRCI+Q & 4.952 & 135.2 & 1.4 & 0.160 & 0.107 & 1.141 \\
\hline & $\operatorname{CCSD}(\mathrm{T})$ & 4.944 & 147.8 & 0.9 & & 0.067 & \\
\hline \multirow[t]{2}{*}{ AVQZ } & MRCI+Q & 4.858 & 146.4 & 1.5 & 0.217 & 0.166 & 1.100 \\
\hline & $\operatorname{CCSD}(\mathrm{T})$ & 4.868 & 158.2 & 1.0 & & 0.125 & \\
\hline \multirow[t]{2}{*}{ AV5Z } & MRCI+Q & 4.838 & 147.8 & 1.7 & 0.225 & 0.179 & 1.099 \\
\hline & $\operatorname{CCSD}(\mathrm{T})$ & 4.852 & 158.0 & 0.9 & & 0.137 & \\
\hline \multirow[t]{2}{*}{ CBS(1) } & MRCI+Q & 4.825 & 149.0 & 1.8 & 0.230 & 0.187 & 1.099 \\
\hline & $\operatorname{CCSD}(\mathrm{T})$ & 4.843 & 157.8 & 0.9 & & 0.144 & \\
\hline \multirow[t]{2}{*}{$\mathrm{CBS}(2)$} & MRCI+Q & 4.815 & 150.2 & 1.7 & 0.234 & 0.193 & 1.099 \\
\hline & $\operatorname{CCSD}(\mathrm{T})$ & 4.836 & 157.7 & 0.8 & & 0.150 & \\
\hline${ }^{3} \Pi_{2}^{d}$ & $\mathrm{MRCI}+\mathrm{Q}$ & 4.782 & 154.0 & 1.2 & 0.250 & 0.155 & 1.120 \\
\hline${ }^{3} \Pi_{1}^{\bar{d}}$ & $\mathrm{MRCI}+\mathrm{Q}$ & 4.847 & 147.8 & 1.7 & 0.218 & 0.231 & 1.077 \\
\hline \multirow[t]{2}{*}{${ }^{3} \Pi_{0}^{d}$} & MRCI+Q & 4.877 & 146.7 & 1.2 & 0.202 & 0.268 & 1.055 \\
\hline & & \multicolumn{6}{|c|}{${ }^{1} \Pi$ state } \\
\hline AVTZ & MRCI+Q & 4.577 & 290.5 & 1.3 & 0.718 & 0.691 & \\
\hline AVQZ & $\mathrm{MRCI}+\mathrm{Q}$ & 4.558 & 295.0 & 1.3 & 0.778 & 0.705 & \\
\hline AV5Z & $\mathrm{MRCI}+\mathrm{Q}$ & 4.551 & 296.3 & 1.3 & 0.790 & 0.713 & \\
\hline CBS(1) & $\mathrm{MRCI}+\mathrm{Q}$ & 4.547 & 297.0 & 1.3 & 0.798 & 0.718 & \\
\hline CBS(2) & $\mathrm{MRCI}+\mathrm{Q}$ & 4.543 & 297.6 & 1.3 & 0.804 & 0.722 & \\
\hline
\end{tabular}

$a$ : the $\omega_{e}$ values are calculated for the ${ }^{202} \mathrm{Hg}^{32} S$ isotopomer.

$b: \Delta T_{e}$ is the difference between the energy of the state and that of the $\mathrm{X}^{1} \Sigma^{+}$ground state, at the same level of calculation.

$c: \Delta E_{a s s}$. is the energy difference between the lowest two asymptotes. The experimental value, averaged on the three fine structure levels, is $1.105 \mathrm{eV}$.

$d$ : Spin-orbit ${ }^{3} \Pi_{\Omega=2,1,0}$ components, deduced from CBS(2) energy values including spin-orbit interactions (see parag. 4.3). 
Table 5: Vibrational energy levels ${ }^{a}\left(\mathrm{in}^{-1}\right.$ ) of the lowest electronic states relative to the minimum energy level $(\mathrm{v}=0)$ of the $\mathrm{X}^{1} \Sigma^{+}$state.

\begin{tabular}{c|cccc}
\hline \hline Level $(\mathrm{v})$ & $\mathrm{E}\left({ }^{3} \Pi_{0}\right)$ & $\mathrm{E}\left({ }^{1} \Sigma_{0}^{+}\right)$ & $\mathrm{E}\left({ }^{3} \Pi_{2}\right)$ & $\mathrm{E}\left({ }^{1} \Pi_{1}\right)$ \\
\hline $0\left(\mathrm{~T}_{0}\right)$ & 2059 & 0 & 1156 & 5794 \\
1 & 2207 & 359 & 1311 & 6089 \\
2 & 2352 & 714 & 1465 & 6382 \\
3 & 2491 & 1065 & 1615 & 6672 \\
4 & 2623 & 1411 & 1759 & 6959 \\
5 & 2747 & 1753 & 1895 & 7243 \\
6 & & 2090 & & 7525 \\
7 & & 2422 & & 7804 \\
8 & & 2750 & & 8080 \\
9 & & 3074 & & \\
10 & & 3393 & \\
11 & & 3706 & \\
\hline \hline
\end{tabular}

(a): Values obtained from the potential energies calculated with MRCI+Q potentials and the CBS(2) extrapolation. 
Figure 1: Potential energy curves, calculated at the MRCI+Q level and AV5Z basis sets, of the electronic states of $\mathrm{HgS}$ correlated to the lowest three asymptotes.

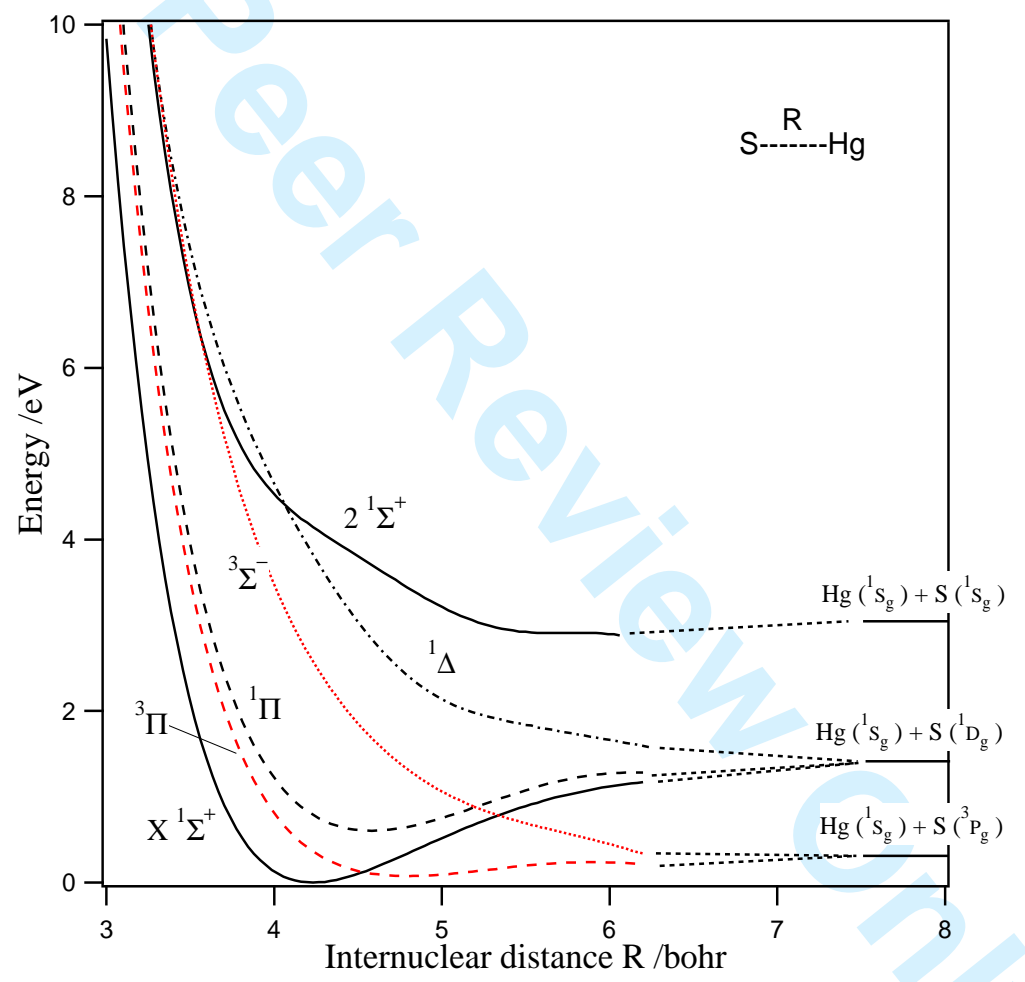


Figure 2: Dipole moment functions, calculated at the MRCI level and AV5Z basis sets, of the low lying electronic states of $\mathrm{HgS}$.

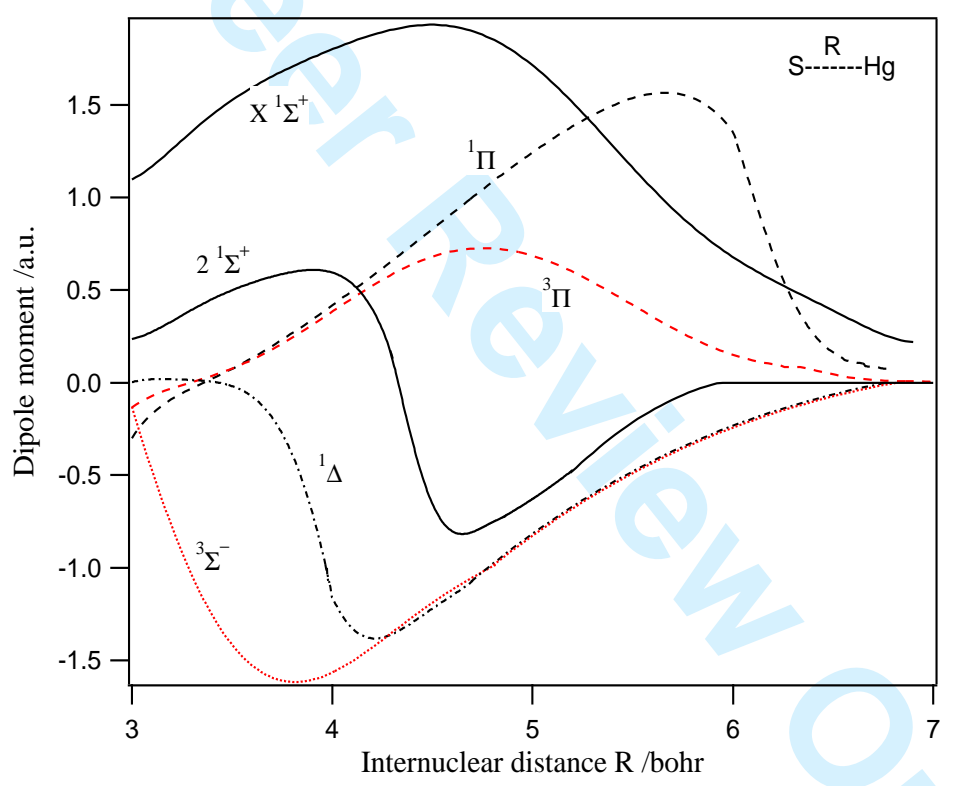


Figure 3: Potential energy curves ${ }^{a}$ of the ${ }^{1} \Sigma^{+}$and ${ }^{3} \Pi$ states of $\mathrm{HgS}$ for different basis sets and with CBS extrapolations.

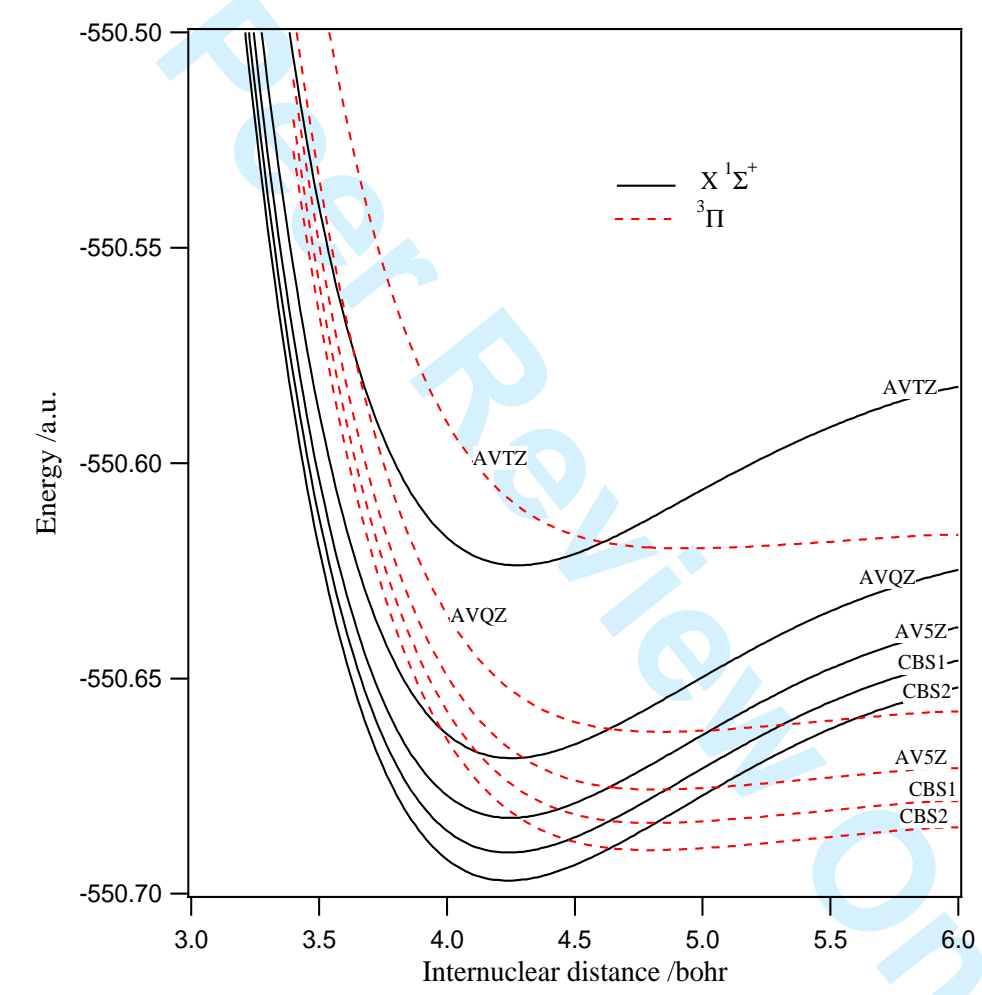

$a$ : (MRCI+Q) energy. Value of the energy minimum of the ${ }^{1} \Sigma^{+}$state with the $\mathrm{CBS}(2)$ extrapolation: $E=-550.6969367$ a.u. 
Figure 4: Evolution of the spin-orbit matrix elements $\left(\right.$ in $\mathrm{cm}^{-1}$ ) between the low lying electronic states ${ }^{a}$.

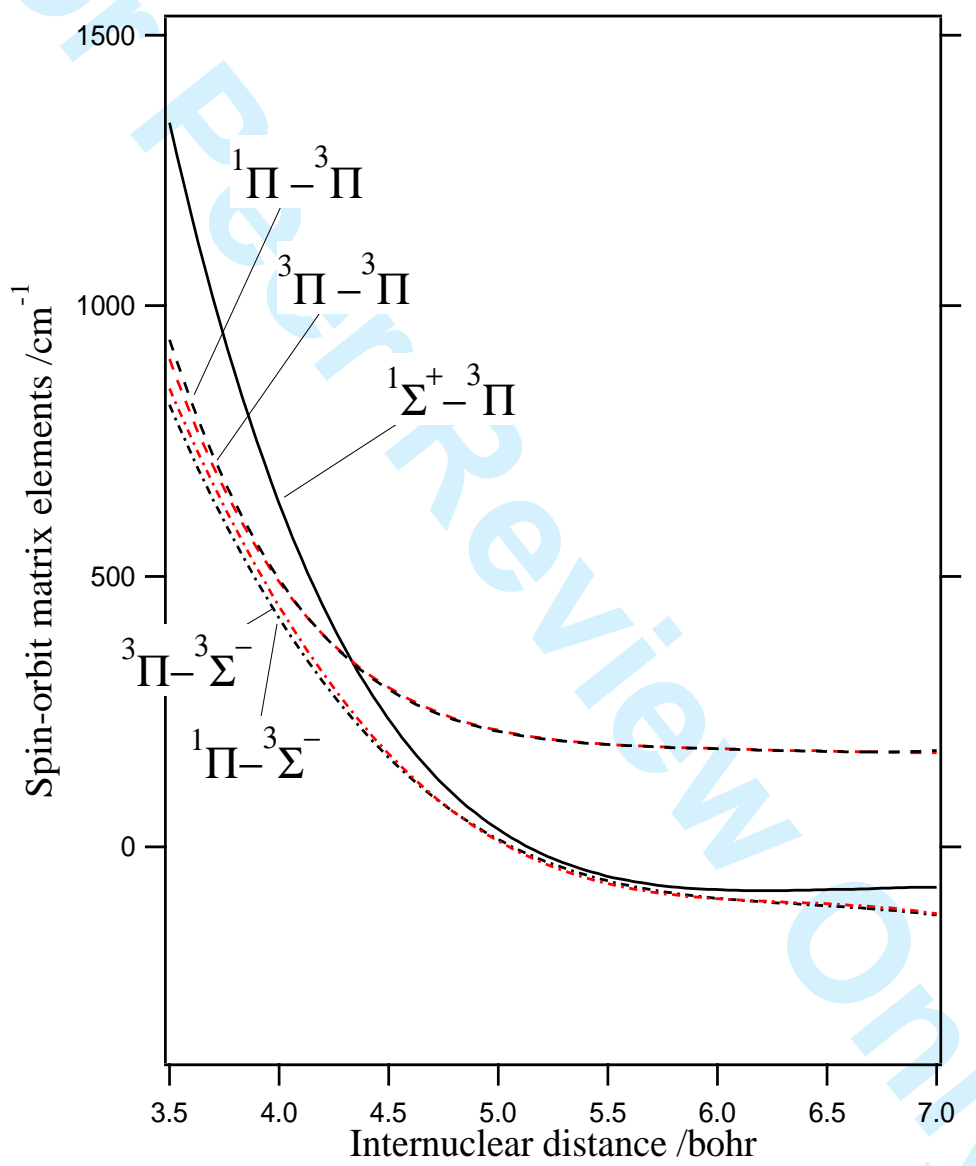

$a$ : Calculated values are available on request. 
Figure 5: Potential energy curves of the ${ }^{1} \Sigma_{\Omega=0}^{+}$and ${ }^{3} \Pi_{\Omega=0,1,2}$ states of $\operatorname{HgS}(\mathrm{CBS}(2)$ extrapolation including spin-orbit interactions).

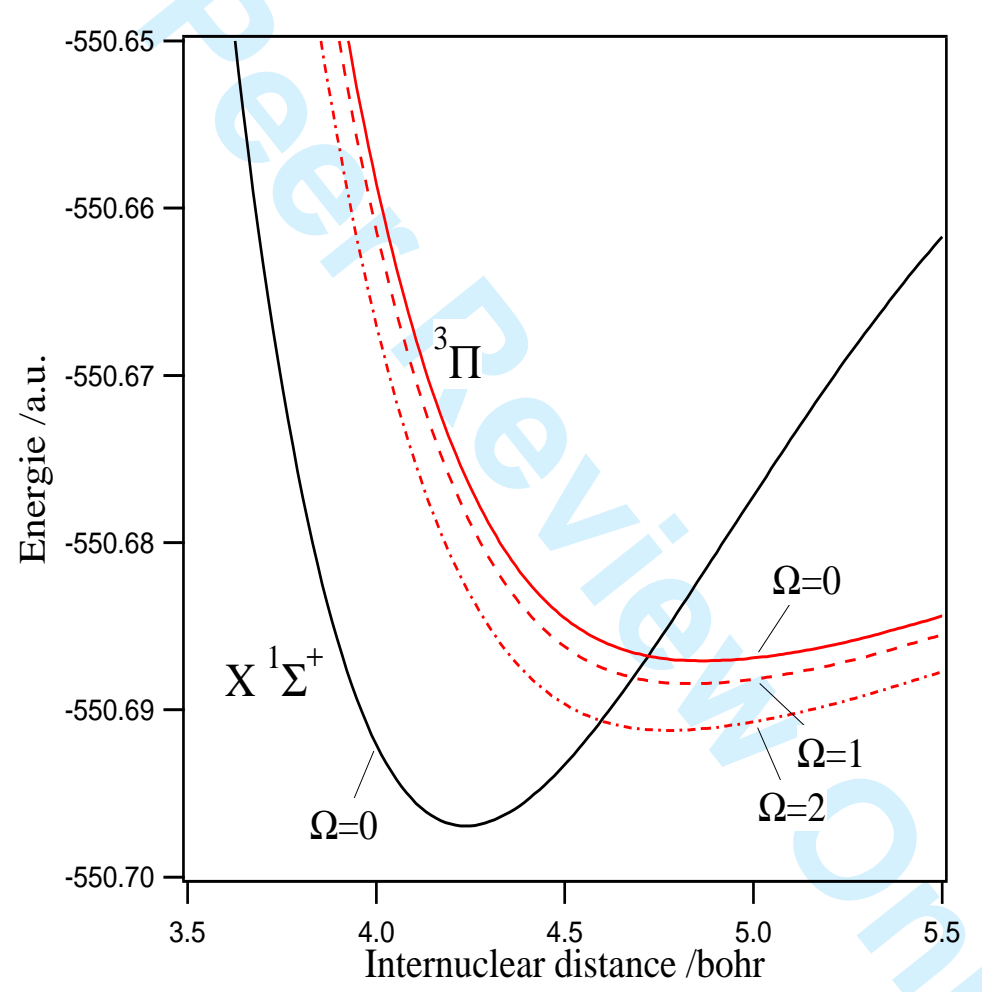




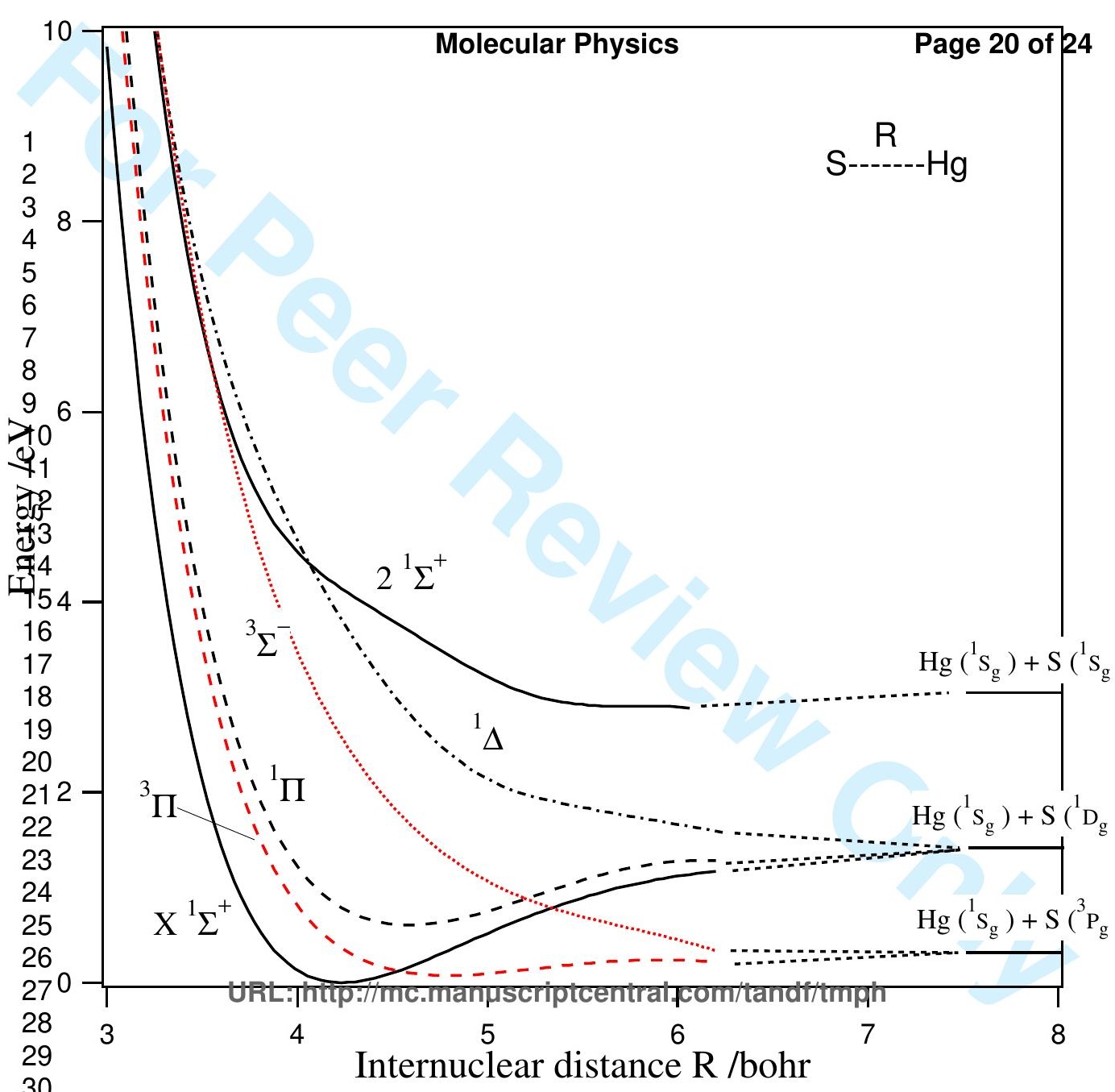




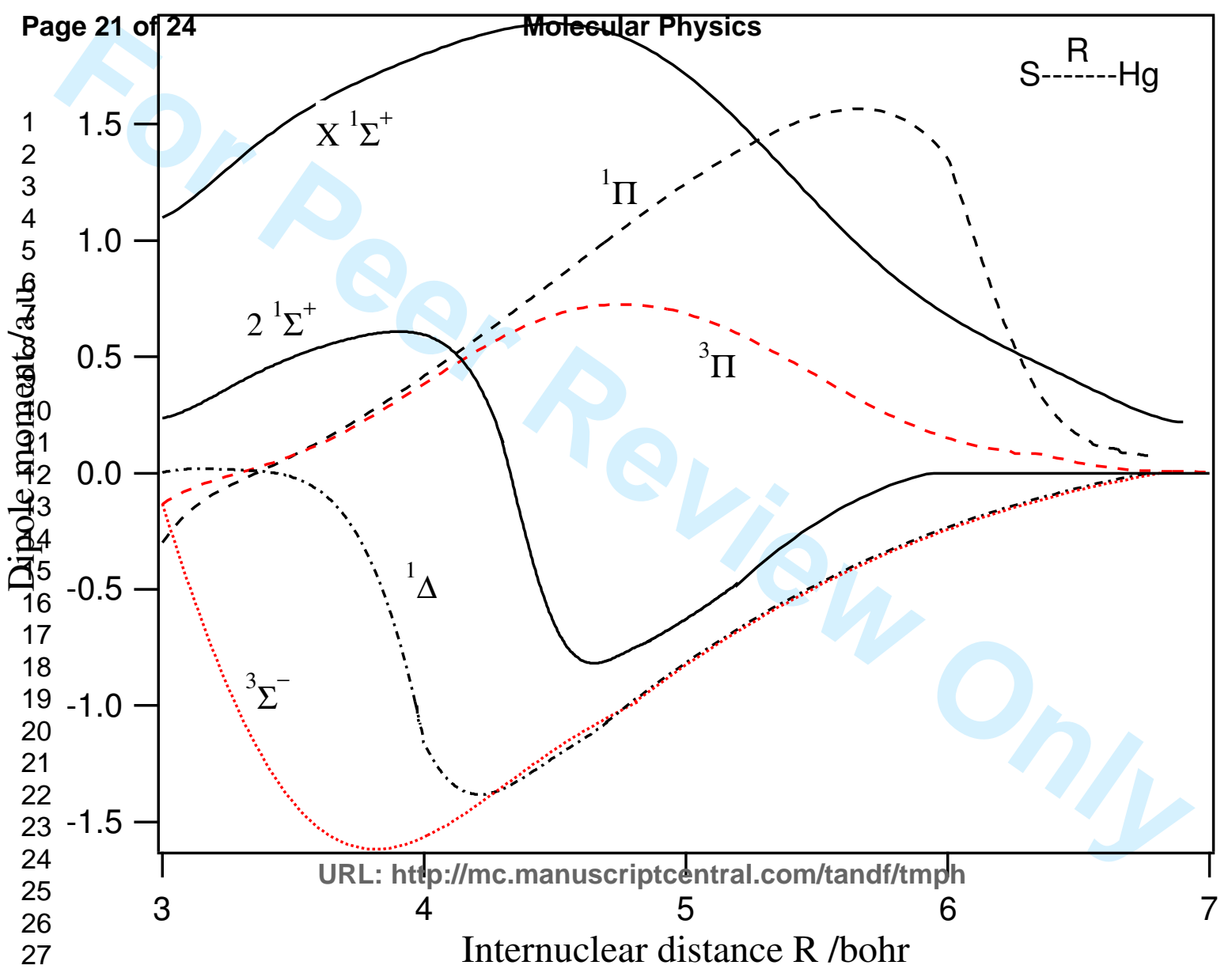




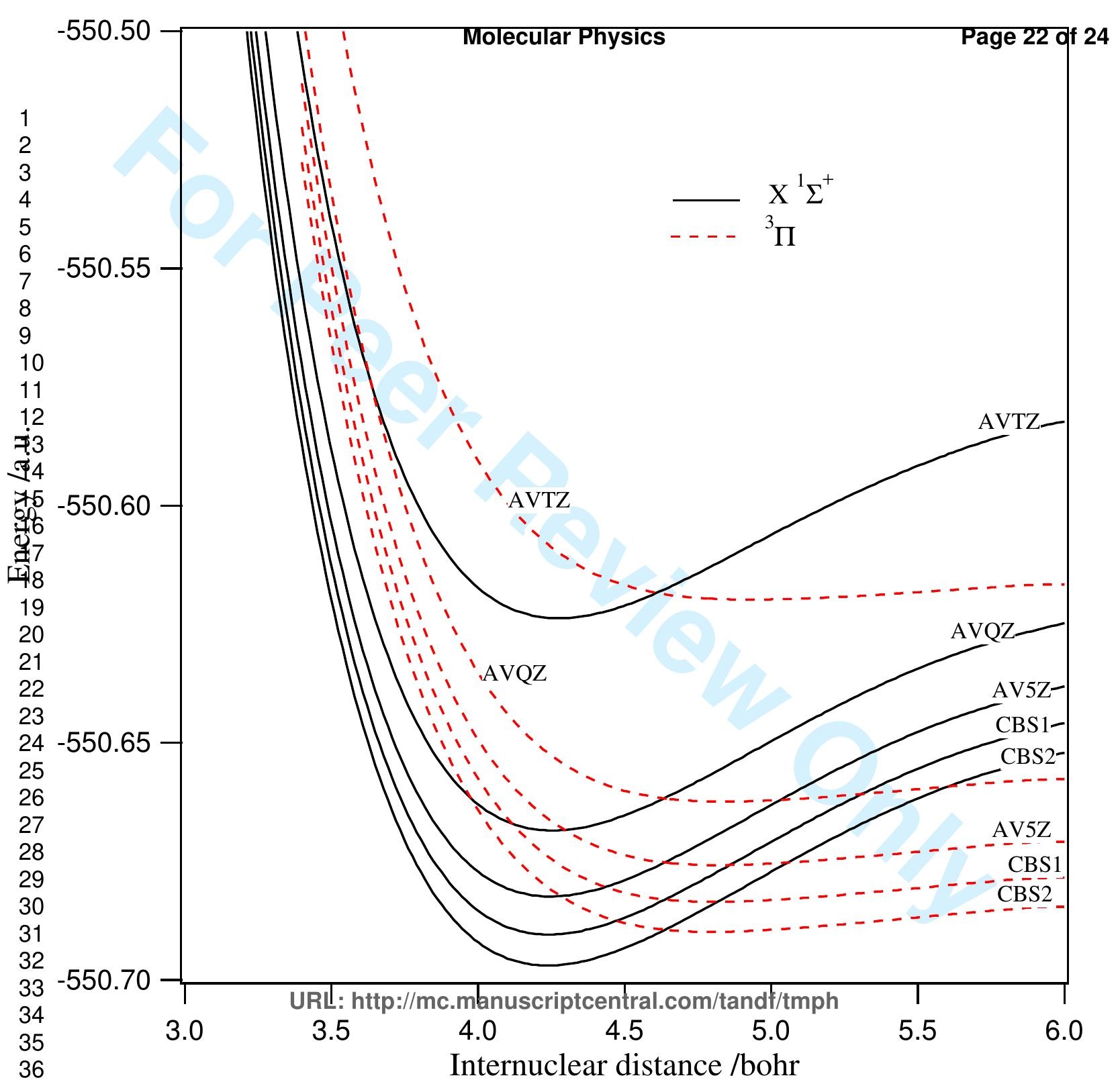




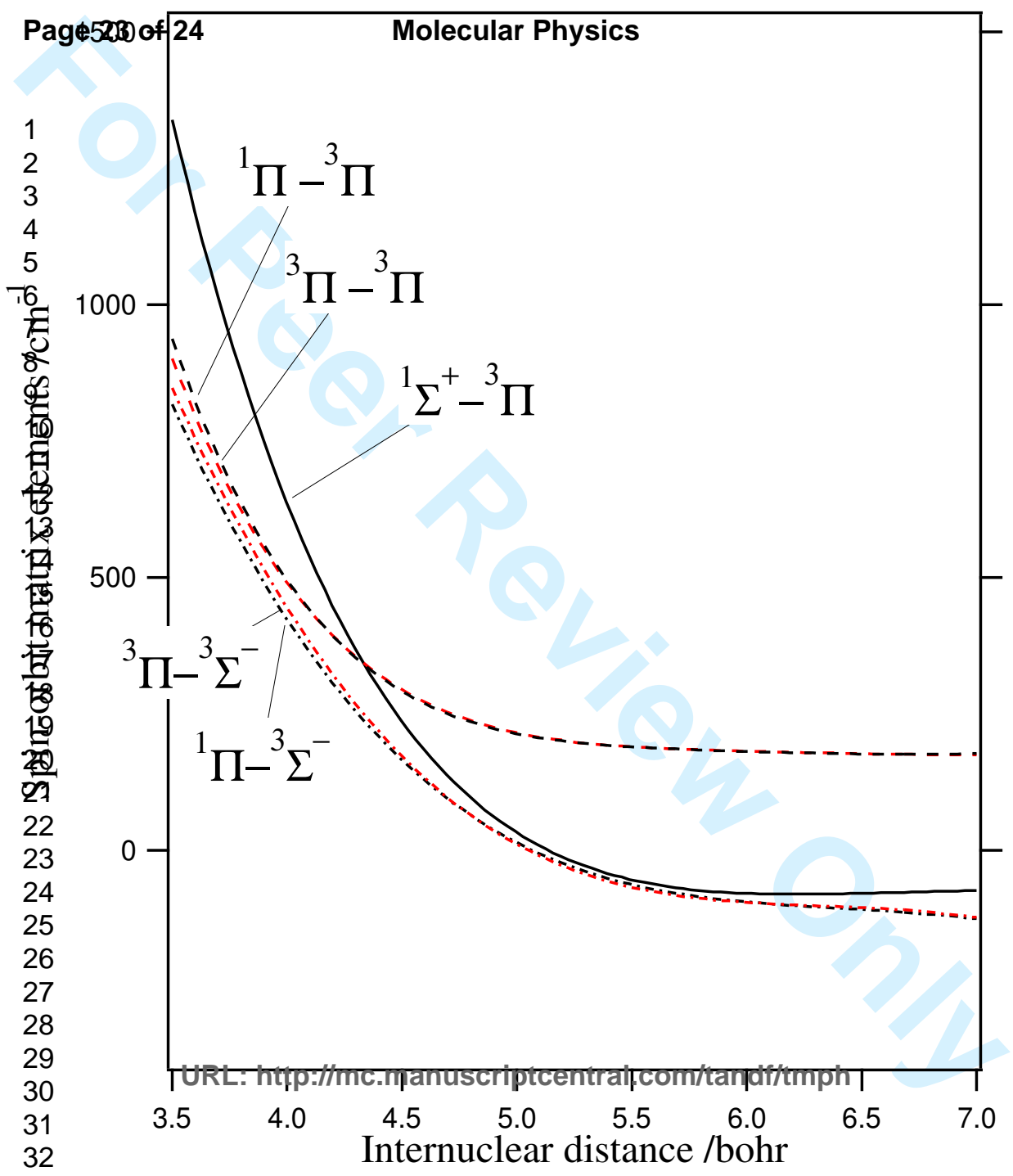


